\title{
Local anaesthetic use among GDPs
}

\author{
A survey of local anaesthetic use among general dental practitioners in the UK attending postgraduate courses \\ on pain control \\ I. P. Corbett, J. C. Ramacciato, F. C. Groppo and J. G. Meechan Br Dent J 2005; 199: 784-787
}

\begin{abstract}
Objective
The aim of this study was to identify which local anaesthetic solutions were used by general dental practitioners in the United Kingdom and to determine selection criteria. In addition, differences in anaesthetic choice between recent graduates ( $\leq 5$ years) and more experienced practitioners were investigated.

Material and methods

Five hundred and six general dental practitioners attending postgraduate courses on pain control in dentistry completed a questionnaire. Participants were asked to indicate year and place of qualification, anaesthetic solutions available in their surgeries and criteria used in the choice of anaesthetic. In addition, the respondents were asked to indicate choice of local anaesthetic in a number of common medical conditions. Questionnaires were distributed and collected immediately prior to the start of the course presentation and participants were not asked to indicate whether the selection decisions were teaching, experience or evidence based. Data were analysed by using the Chi-square test.

Results

Lidocaine with epinephrine was the most widely available solution among this group of practitioners (94\%), the second most common solution was prilocaine with felypressin (74\%). The majority of practitioners had two or more solutions available. Practitioners who qualified within the last five years (14\%) were more likely to have articaine available, the most recently introduced local anaesthetic into the UK ( $p=0.04$, one degree of freedom). Common medical conditions lead to a modification in anaesthetic selection: the use of prilocaine/felypressin increases in the majority of circumstances, although it is avoided in pregnant females by recent graduates. Conclusions

Lidocaine/epinephrine continues to be the most common anaesthetic solution used by this group of UK general practitioners. The primary criterion for selection of an anaesthetic agent was perceived efficacy. Prilocaine/felypressin is commonly selected as an alternative solution in the presence of common medical conditions.
\end{abstract}

\section{IN BRIEF}

- Reviews evidence with regard to anaesthetic selection in common medical conditions.

- Indicates current practice within the dental population.

- Emphasises the need for evidence-based practice.

\section{COMMENT}

This interesting study uses a questionnaire survey to report which local anaesthetics are used by 506 UK dentists and the reasons for their selection. It investigates the influence of the patient's general health on choice of local anaesthetic and compares responses from dentists recently graduated with those of more than five years' experience. This paper illustrates very well the gap between clinical practice and research evidence and highlights the issue of decision making as we move towards more evidence based clinical practice.

The most commonly used local anaesthetic was lidocaine with epinephrine with dentists giving anaesthetic efficacy as the reason for their choice. Most practitioners also used prilocaine with felypressin. Articaine was more commonly used by recent graduates.

Prilocaine with felypressin was used rather than lidocaine with adrenaline for patients with a history of recent myocardial infarction. A plain solution such as $4 \%$ prilocaine would be preferable in this situation although only $7 \%$ of the sample used this. Prilocaine with felypressin was also used for hypertensive patients even though the changes in systolic and diastolic pressure when using an epinephrine-containing solution are clinically insignificant. Newer graduates were more likely to use lidocaine with epinephrine for hypertensive patients than those graduated for longer.

Some practitioners selected prilocaine with felypressin rather than lidocaine with adrenaline for asthmatic patients although neither is contraindicated. These practitioners tended to be in the 'more experienced' group. Lidocaine with epinephrine was also dropped in favour of prilocaine with felypressin for patients with diabetes although any theoretical risk of increasing blood glucose is not clinically significant.

The results suggest that dentists are less influenced by the scientific evidence than other factors when considering choice of local anaesthesia. Factors may include the opinions of others, previous education, perceived legal pressure and marketing. This paper may therefore encourage dentists to consider their choice and question influencing factors such as pharmaceutical advertisements. Perhaps more importantly, this paper provides a challenge for academics to provide the best evidence of local anaesthetic efficacy and safety for practitioners that is easy to access so that they may keep up to date.

P. Coulthard, Oral and Maxillofacial Surgery, School of Dentistry, The University of Manchester doi: $10.1038 /$ sj.bdj.4813038 